\title{
Ongoing Clinical Trials
}

Section Editor: S. Galandiuk, MD, Louisville

Dig Surg 2007;24:234-242

Published online: May 30, 2007

This section provides information for researchers on clinical trials being in progress in their field throughout the world. The list of trials described herein is by no means inclusive, and the publisher is not responsible for any data given.

Please use the special questionnaire at the end of this section to submit information on a new trial.

The Ongoing Clinical Trials are only available online, free of charge, under www.karger.com/dsu_issues

Oncology

Surgical Infection / Sepsis

Miscellaneous

Questionnaire for Trial Submission 


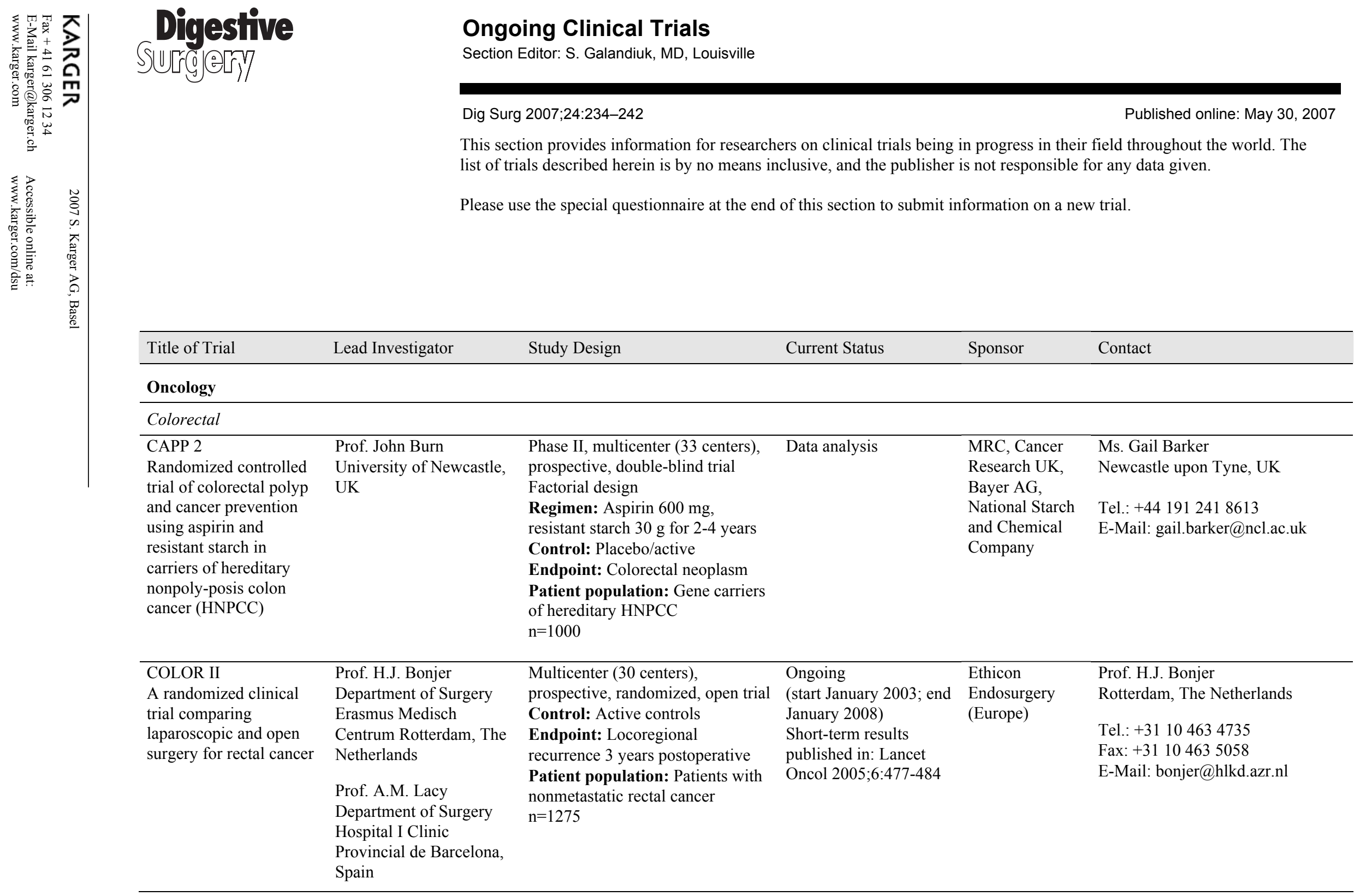


Ongoing Clinical Trials (continued)

\begin{tabular}{|c|c|c|c|c|c|}
\hline Title of Trial & Lead Investigator & Study Design & Current Status & Sponsor & Contact \\
\hline $\begin{array}{l}\text { DaCHS - Prevention of } \\
\text { colorectal carcinoma: } \\
\text { The role of screening }\end{array}$ & $\begin{array}{l}\text { Prof. Dr. H. Brenner } \\
\text { Deutsches Zentrum für } \\
\text { Alternsforschung, } \\
\text { Heidelberg, Germany }\end{array}$ & $\begin{array}{l}\text { Independent, case-control trial } \\
\mathrm{n}=1500\end{array}$ & $\begin{array}{l}\text { Ongoing (start January } \\
\text { 2003; end of recruit- } \\
\text { ment December 2007) }\end{array}$ & $\begin{array}{l}\text { DKFZ } \\
\text { (Deutsches } \\
\text { Krebsforschungs- } \\
\text { zentrum) }\end{array}$ & $\begin{array}{l}\text { Dr. Christoph Seiler, MSc } \\
\text { Heidelberg, Germany } \\
\text { Tel.: +496221566986 } \\
\text { Fax: +49 6221566988 } \\
\text { E-Mail: christoph_seiler@med.uni- } \\
\text { heidelberg.de } \\
\text { www.sdgc.de }\end{array}$ \\
\hline $\begin{array}{l}\text { Colon-J-pouch versus } \\
\text { transverse coloplasty } \\
\text { pouch: A randomized } \\
\text { controlled trial com- } \\
\text { paring functional results } \\
\text { after rectum resection } \\
\text { and different recon- } \\
\text { structions } \\
\text { ISRCTN No.: } 78983587\end{array}$ & $\begin{array}{l}\text { PD Dr. Kaspar Z'graggen } \\
\text { Klinik Beau-Site, Berne, } \\
\text { Switzerland }\end{array}$ & $\begin{array}{l}\text { Multicenter, prospective, ran- } \\
\text { domized, open trial } \\
\text { Control: Comparison of different } \\
\text { techniques } \\
\text { Endpoint: Morbidity, function and } \\
\text { mortality during a follow-up period } \\
\text { of } 24 \text { months } \\
\text { Patient population: Patients } \\
\text { showing deep rectum pathology } \\
\text { with planned RO-surgery } \\
n=150\end{array}$ & Follow-up & & $\begin{array}{l}\text { Dr. Christoph Seiler, MSc } \\
\text { Heidelberg, Germany } \\
\text { Tel.: +496221566986 } \\
\text { Fax: +496221566988 } \\
\text { E-Mail: christoph_seiler@med.uni- } \\
\text { heidelberg.de } \\
\text { www.sdgc.de }\end{array}$ \\
\hline $\begin{array}{l}\text { ROMIC: Role of ovarian } \\
\text { metastasis in colorectal } \\
\text { cancer }\end{array}$ & $\begin{array}{l}\text { R.M.H. Roumen, MD } \\
\text { Maxima Medical Center } \\
\text { Department of Surgery } \\
\text { Veldhoven, the } \\
\text { Netherlands }\end{array}$ & $\begin{array}{l}\text { Multicenter (12 centers), } \\
\text { prospective, unblinded } \\
\text { (oophorectomy) trial } \\
\text { Control: Patients who do not } \\
\text { undergo oophorectomy } \\
\text { Endpoint: } 3 \text {-year disease-free } \\
\text { survival } \\
\text { Patient population: Postmeno- } \\
\text { pausal women with colorectal } \\
\text { cancer without evidence of } \\
\text { metastatic disease } \\
\text { n=1000 } \\
\text { Additional information: } \\
\text { Incidence of micrometastases in } \\
\text { ovaria of colorectal cancer patients } \\
\text { and prevention of future } \\
\text { Krukenberg tumors }\end{array}$ & $\begin{array}{l}\text { Ongoing (start 2003; } \\
\text { end 2007) }\end{array}$ & & $\begin{array}{l}\text { R.M.H. Roumen, MD } \\
\text { Veldhoven, the Netherlands } \\
\text { Tel.: +31408888556 } \\
\text { Fax: +31408888565 } \\
\text { E-Mail: r.roumen@mmc.nl }\end{array}$ \\
\hline
\end{tabular}


Ongoing Clinical Trials (continued)

\begin{tabular}{|c|c|c|c|c|c|}
\hline Title of Trial & Lead Investigator & Study Design & Current Status & Sponsor & Contact \\
\hline $\begin{array}{l}\text { Influence of two } \\
\text { different resection } \\
\text { techniques of liver }\end{array}$ & $\begin{array}{l}\text { Jürgen Weitz, MD } \\
\text { Department of Surgery, } \\
\text { University of Heidelberg, }\end{array}$ & $\begin{array}{l}\text { Multicenter ( } 3 \text { centers), } \\
\text { prospective open trial } \\
\text { Control: Comparison of two }\end{array}$ & Ongoing & $\begin{array}{l}\text { Prof. M.W. } \\
\text { Büchler }\end{array}$ & $\begin{array}{l}\text { Dr. Christoph Seiler, MSc } \\
\text { Heidelberg, Germany }\end{array}$ \\
\hline
\end{tabular}

metastases from

colorectal cancer on

hematogenous tumor

cell dissemination

ISRCTN No.: 45066244
Germany

techniques

Endpoint: Tumor cell detection in

blood samples

Patient population: Patients with

liver metastases of a colorectal

cancer
Tel.: +496221566986

Fax: +496221566988

E-Mail: christoph_seiler@med.uni-

heidelberg.de

www.sdgc.de

Pancreatic

Primary resection vs.

neoadjuvant chemo-

Prof. Dr. W. Hohenberger Multicenter, prospective,

nonrandomized trial

Ongoing (start June

resection for locally

Erlangen, Germany

Patient population: Patients with

resectable or potentially

resectable in pancreatic

Prof. Dr. G. Grabenbauer the pancreatic head

potentially resectable carcinom

$\mathrm{n}=254$ (127 per arm)

carcinoma without $\quad$ Erlangen, Germany

Additional information: Pilo

distant metastasis

project finished, new trial with

gemcitabine and cis-platin as

radiosensitizers

$\begin{array}{ll}\text { Ongoing (start June } & \text { PD Dr. T. Meyer } \\ \text { 2003) } & \text { Erlangen, Germany }\end{array}$

Tel : +4991318533296

Fax: +4991318536595

E-Mail:

Thomas.meyer@chir.imed.uni-

erlangen.de

PD Dr. T. Brunner

Erlangen, Germany

E-Mail:

thomas.brunner@strahlen.imed.unierlangen.de

ESPAC-3(v2)

Adjuvant chemo-

therapies in resectable

Prof. J.P. Neoptolemos

Department of Surgery,

International multicenter (154

centers) trial; patients with

Ongoing

pancreatic ductal adenocarcinoma

randomized into one of two groups

(gemcitabine; 5-FU and folinic

acid) and patients with ampullary

or other tumors of the pancreas

randomized into one of three

groups (gemcitabine; 5-FU and

folinic acid, and observation)

Endpoint: Overall survival

Patient population: Patients with

resected pancreatic cancer

$\mathrm{n}=1030$
Cancer Research Mrs. Emily Owen, Coordinator

Liverpool, UK

Tel : +44 1517948932

Fax: +44 1517948930

E-Mail: e.owen@liverpool.ac.uk 


\begin{tabular}{|c|c|c|c|c|c|}
\hline \\
\hline Title of Trial & Lead Investigator & Study Design & Current Status & Sponsor & Contact \\
\hline $\begin{array}{l}\text { GEM-CAP } \\
\text { Trial comparing } \\
\text { gemcitabine alone or in } \\
\text { combination with } \\
\text { capecitabine for the } \\
\text { treatment of patients } \\
\text { with advanced } \\
\text { pancreatic ductal } \\
\text { adenocarcinoma }\end{array}$ & $\begin{array}{l}\text { Professor J.P. } \\
\text { Neoptolemos } \\
\text { Department of Surgery, } \\
\text { University of Liverpool, } \\
\text { UK }\end{array}$ & $\begin{array}{l}\text { Phase III, mulicenter, randomized } \\
\text { trial } \\
\text { Regimen: } \\
\text { Patients treated with gemcitabine } \\
\text { alone or a combination of } \\
\text { gemcitabine and capecitabine for } \\
12 \text { weeks; those responding to } \\
\text { treatment or with stable disease } \\
\text { receive a further } 12 \text { weeks of } \\
\text { treatment } \\
\text { Primary endpoint: One-year } \\
\text { survival } \\
\text { Secondary endpoint: Quality of } \\
\text { life; median and two-year survival; } \\
\text { toxicity; objective response rate } \\
\text { Patient population: Patients with } \\
\text { histological or cytological evidence } \\
\text { of locally advanced / metastatic } \\
\text { carcinoma of the pancreas not } \\
\text { amenable to curative surgery / } \\
\text { radiotherapy; } n=508\end{array}$ & $\begin{array}{l}\text { Ongoing } \\
\text { (closed to recruitment as } \\
\text { target accrual has been } \\
\text { reached) }\end{array}$ & & $\begin{array}{l}\text { Ms. Claire Davies, Coordinator } \\
\text { Liverpool, UK } \\
\text { Tel.: +44 } 1517948933 \\
\text { Fax: +44 } 1517948930 \\
\text { E-Mail: cldavies@liverpool.ac.uk }\end{array}$ \\
\hline \multicolumn{6}{|l|}{ Miscellaneous } \\
\hline $\begin{array}{l}\text { Multicenter randomized } \\
\text { trial for laser-induced } \\
\text { thermotherapy of colo- } \\
\text { rectal liver metastases }\end{array}$ & $\begin{array}{l}\text { Priv. Doz. C.-T. Germer } \\
\text { Department of Surgery, } \\
\text { University Hospital BF } \\
\text { Berlin, Germany }\end{array}$ & $\begin{array}{l}\text { Phase III, multicenter (6 centers), } \\
\text { randomized trial } \\
\text { Control: Surgical liver resection } \\
\text { Endpoint: Patient survival, quality } \\
\text { of life/mortality } \\
\text { Patient population: Patients with } \\
4 \text { or fewer liver metastases, } \\
\text { diameter } 4 \text { cm or smaller and with } \\
\text { no signs of extrahepatic malignant } \\
\text { disease } \\
\mathrm{n}=400\end{array}$ & $\begin{array}{l}\text { Recruitment phase (start } \\
\text { August 2000; } \\
\text { end July 2007) }\end{array}$ & BMBF & $\begin{array}{l}\text { Prov. Doz. C.-T. Germer } \\
\text { Berlin, Germany } \\
\text { Tel.: +49 } 3084452543 \\
\text { Fax: +49 } 3084452740 \\
\text { E-Mail: germer@ukbf.fu-berlin.de }\end{array}$ \\
\hline
\end{tabular}




\section{Surgical Infection / Sepsis}

\section{PANTER}

Prof. H.G. Gooszen

Multicenter (20 centers of the

Dutch Acute Pancreatitis Study

Minimally invasive Head, Department of

'step-up approach' vs. Surgery

maximal necrosectomy University Medical

in patients with acute Center Utrecht, The

necrotizing pancreatitis Netherlands

Chairman Dutch Acute

Pancreatitis Study Grou

Group), prospective, non-blinded,

active-controlled trial

Patients are randomized between

A) Maximal necrosectomy by

laparotomy with continuous

postoperative lavage and B) CT-

guided percutaneous or endoscopic

transgastric drainage, if necessary

followed by videoscopic assisted

retroperitoneal debridement

\section{(VARD)}

Endpoint: Total mortality and

major morbidity

Patient population: Patients with

(suspected) infected necrotizing

pancreatitis, preferably $>$ days after

onset of symptoms

$\mathrm{n}=88$

\begin{tabular}{|c|c|c|c|c|c|}
\hline $\begin{array}{l}\text { PROPATRIA } \\
\text { Multicenter, } \\
\text { randomized, blinded, } \\
\text { placebo-controlled trial } \\
\text { of probiotic prophylaxis } \\
\text { in predicted severe acute } \\
\text { pancreatitis }\end{array}$ & $\begin{array}{l}\text { Prof. H.G. Gooszen } \\
\text { Head, Department of } \\
\text { Surgery } \\
\text { University Medical } \\
\text { Center Utrecht, The } \\
\text { Netherlands } \\
\text { Chairman Dutch Acute } \\
\text { Pancreatitis Study Group }\end{array}$ & $\begin{array}{l}\text { Investigator-initiated, multicenter, } \\
\text { double-blind, placebo-controlled } \\
\text { trial } \\
\text { Regimen: Patients randomly } \\
\text { assigned to receive either live } \\
\text { multispecies probiotics ( } 6 \text { strains, } \\
\text { Ecologica } 641 \text { ) or placebo for } 4 \\
\text { weeks by nasojejunal tube. } \\
\text { Treatment started within } 72 \text { hours } \\
\text { of onset of abdominal pain } \\
\text { Patient Population: Predicted } \\
\text { acute severe pancreatitis }\end{array}$ & $\begin{array}{l}\text { Ongoing (start March } \\
\text { 2004; estimated end } \\
\text { 2007) }\end{array}$ & $\begin{array}{l}\text { University } \\
\text { Medical Center, } \\
\text { Utrecht. } \\
\text { Funded by } \\
\text { Senter, an } \\
\text { agency of the } \\
\text { Ministry of } \\
\text { Economic } \\
\text { Affairs }\end{array}$ & $\begin{array}{l}\text { Marc G.H. Besselink, MD } \\
\text { Utrecht, The Netherlands } \\
\text { Tel.: +31 } 302508074 \\
\text { Fax: +31 } 302541944 \\
\text { E-Mail: } \\
\text { m.besselink@umcutrecht.nl }\end{array}$ \\
\hline
\end{tabular}
Ongoing (start January The Netherlands Prof. H.G. Gooszen
2006; end January Organization for Utrecht, The Netherlands
2009) Health Research
protocol submitted to (ZonMw, grant E-Mail: h.gooszen@umcutrecht.nl
BioMed Central, no. 945-06-910) 


\section{Miscellaneous}

Double-blind

randomized controlled

trial to investigate the

efficacy of Antox and

MGCT for the treatment

of hereditary pancreatitis

and idiopathic chronic

pancreatitis
Phase III, double-blind, placebo-

controlled randomized trial

Regimen: Antioxidants for 1 year

Control: Placebo

Primary endpoint: Reduction in

the number of days of pancreatic

pain

Secondary endpoint: Analgesic

use; hospital admissions; quality of

\section{life scores}

Patient population: Patients $(5-40$

years old) with hereditary pancre-

atitis or ideopathic chronic pancre-

atitis, registered with EUROPAC,

having characteristic pain that is

either intermittent or continuous
Recruitment

(start 2006)

Single center, prospective, patient- Follow-up

pain outcome in patients Department of Surgery blind trial

with vertical and trans-

verse abdominal

niversity of Heid

Germany

incision: A randomized

controlled equivalence

trial

ISRCTN Nr.: 60734227

INSECT: Interrupted or

continuous slowly

absorbable suture

evaluation of abdo

University of Heidelberg

closure techniques
Prof. M.W. Büchler

Department of Surgery

Germany

\section{Control: Comparison of thre}

techniques

Endpoint: Frequency of

abdominal hernias after 12 months

and 3 years

Patient population: Patients who

are planned to undergo an elective

abdominal operation
Michael G.T. Raraty, MB, BS,

PhD, FRCS

EUROPAC Study Coordinator Liverpool, UK

Tel.: +44 1517064170

Fax: +44 1517065826

E-Mail: mraraty@liv.ac.uk

europac@liv.ac.uk 
randomized controlled University of Heidelbers
LAPCON: Total laparos- Prof. M.W. Büchler copic versus conven- Department of Surgery tional ileoanal pouch University of Heidelberg procedure: a randomized Germany controlled tria
Single center, prospective, open trial

Control: Laparoscopic versus

procedure

Endpoint: Intraoperative blood loss

Patient population: Patients with

familiar polyposis or ulcerative

colitis, scheduled for elective

proctocolectomy with ileoanal

pouch conventional ileoanal pouch

Ongoing

g

Prof. M.W.
Büchler

Multicenter, prospective, open trial Ongoing

$\begin{array}{lll}\text { CLIVIT: Clips vs. } & \text { Prof. M.W. Büchler } & \text { Multicenter, prospective, open } \\ \text { Ligatures. A multicenter } & \text { Department of Surgery } & \text { Control: Comparison of two } \\ \text { randomized controlled } & \text { University of Heidelberg } & \text { techniques to control vessels i }\end{array}$

BBD-Aesculap trial

thyroid surgery

Endpoint: Time of resection

are planned to undergo an elective

bilateral thyroid resection

Open, randomized

multicenter, phase IIIb

study for 5 years to

assess long-term effi-

ciency and tolerability of

esomeprazole compared

to laparoscopic anti-

reflux surgery in adult

subjects with chronic

gastroesophageal reflux

disease

Mesh plug versus

Lichtenstein, prospectiv

and randomized study

Lars Lundell, MD

Sahlgrenska University

Hospital

Gothenburg, Sweden

Phase IIIb, multicenter (60

centers), prospective, open trial

Control: Surgery

Regimen: 20-40 mg esomeprazole

for 5 years

Endpoint: Time to treatment

failure

Patient population: Patients

suffering from confirmed gastro-

esophageal reflux disease for more

than 6 months

$\mathrm{n}=550$

Phase III, multicenter, prospective,

Dr. A. Wildisen

single-blind trial

Endpoint: Hernia recurrence
Patient population: Patients who

Heidelberg, Germany

Tel.: +496221566986

Fax: +49 6221566988

E-Mail:

christoph_seiler@med.uni-

heidelberg.de

www.sdgc.de

Dr. Christoph Seiler, MSc

Heidelberg, Germany

Tel.: +496221566986

Fax: +49 6221566988

E-Mail:

christoph_seiler@med.uni-

heidelberg.de

www.sdgc.de

$\mathrm{n}=900$
Ongoing

(end 2007)
Dr. A. Wildisen

Sursee-Wolhusen, Switzerland
Tel.: +41419264155 


\begin{tabular}{|c|c|c|c|c|}
\hline & & & & \\
\hline $\begin{array}{l}\text { A phase III, double- } \\
\text { blind, randomized, } \\
\text { parallel-group, placebo- } \\
\text { controlled study of } \\
\text { intravenous (IV) } \\
\text { methylnaltrexone } \\
\text { bromide (MNTX) in the } \\
\text { treatment of post- } \\
\text { operativeiIleus (POI) }\end{array}$ & $\begin{array}{l}\text { Phase III, multicenter trial ( } 75-90 \\
\text { centers worldwide) } \\
\text { Control: Placebo } \\
\text { Regimen: IV MNTX } 24 \mathrm{mg} \text {, } \\
\text { MNTX } 12 \mathrm{mg} \text {, or placebo for up to } \\
10 \text { days } \\
\text { Endpoint: Length of time from } \\
\text { end of surgery to first bowel } \\
\text { movement } \\
\text { Patient Population: Patients } \\
\text { scheduled for a segmental } \\
\text { colectomy via open laparotomy } \\
\text { with general anesthesia }\end{array}$ & Ongoing & $\begin{array}{l}\text { Progenics } \\
\text { Pharmaceutical, } \\
\text { Inc. }\end{array}$ & $\begin{array}{l}\text { Anna Williford, RN } \\
\text { Louisville, Ky., USA } \\
\text { Tel.: +1 } 5025830880 \\
\text { Fax: +1 } 5025852988 \\
\text { E-Mail: } \\
\text { aowill01@gwise.louisville.edu }\end{array}$ \\
\hline
\end{tabular}


Digestive

surgergery

\section{Questionnaire}

\section{for Trial Submission}

To submit information on a clinical trial for publication in the 'Ongoing Clinical Trials' section, please complete this short questionnaire.

\section{1) Title of study (in full)}

\section{2) Lead investigator}

Title:

Name:

Affiliation:

\section{3) Field of study}

Please indicate one or more of the following categories:

$\square$ Oncology $\square$ Inflammatory Bowel Disease

$\square$ Motility $\square$ Surgical Infection/Sepsis

$\square$ Miscellaneous

Other (please specify):

\section{4) Study phase (drug trials only)}

Please indicate one of the following categories:

$\square$ Phase I $\square$ Phase II $\square$ Phase III $\square$ Phase IV

$\square$ Independent (investigator initiated)

\section{5) Study design}

$\square$ Multicenter $\square$ Single center (please indicate as appropriate) If multicenter, please state how many centers are involved:

$\square$ Prospective $\square$ Retrospective (please indicate as appropriate)

If prospective, level of randomization (e.g. single blind, double blind, investigator-blind):

Controls (i.e. placebo-, active-, etc.):

Cross-over design (one-way, two-way, etc.):

Duration of therapy (if applicable):

\section{KARGER}

Dosing regimen (if applicable):

Primary study endpoint:

Other information on study design (please include any other details that might be relevant):

\section{6) Patients}

Number of patients (planned):

Patient population (e.g. patients with mild UC previously controlled on...):

\section{7) Current status of study}

Start date:

Estimated end date:

Status (please indicate as appropriate):

$\begin{array}{ll}\square \text { Development } & \square \text { Recruitment } \quad \square \text { Ongoing } \\ \square \text { LPO } & \square \text { Analysis in progress }\end{array}$

$\square$ LPO $\quad \square$ Analysis in progress

\section{Publication}

Abstract submitted to:

Manuscript submitted to:

\section{8) Sponsor}

\section{9) Contact for further information:}

Name:

Address:

Telephone:

Fax:

E-Mail:

Please return the completed questionnaire as soon as possible to the Section Editor:

Susan Galandiuk, MD

Department of Surgery

School of Medicine

University of Louisville

Louisville, KY 40292, USA

Tel.: +1 5028525442

Fax: +1 5028528915 or contact:

S. Karger AG

Attn.: Ms. Yvonne Rebmann

Allschwilerstrasse 10

PO Box

CH-4009 Basel

Tel.: +41613061351

Fax: +41613061234

E-Mail: y.rebmann@karger.ch 